\title{
A spherical joint robotic end-effector for the Expanded Endoscopic Endonasal Approach
}

\author{
Emmanouil Dimitrakakis ${ }^{a}$, George Dwyer $^{a}$, Lukas Lindenroth $^{a}$, Petros Giataganas $^{b}$, Neil L. Dorward ${ }^{c}$, \\ Hani J. Marcus ${ }^{a, c}$, and Danail Stoyanov ${ }^{a}$ \\ ${ }^{a}$ Wellcome/EPSRC Centre for Surgical and Interventional Sciences (WEISS), Charles Bell House, 43-45 Foley Street, \\ University College London, London, UK, W1W $7 T S$ \\ E-mail: e.dimitrakakis@ucl.ac.uk \\ ${ }^{b}$ Digital Surgery Limited, London, UK \\ ${ }^{c}$ National Hospital for Neurology and Neurosurgery, London, UK
}

The endonasal transsphenoidal approach allows surgeons to access the pituitary gland through the natural orifice of the nose. Recently, surgeons have also described an Expanded Endoscopic Endonasal Approach (EEEA) for the treatment of other tumours around the base of the brain. However, operating in this way with non-articulated tools is technically very difficult and not widely adopted. The goal of this study is to develop an articulated end-effector for a novel handheld robotic tool for the EEEA. We present a design and implementation of a $3.6 \mathrm{~mm}$ diameter, three degrees-of-freedom, tendon-driven robotic end-effector that, contrary to rigid instruments which operate under fulcrum, will give the surgeon the ability to reach areas on the surface of the brain that were previously inaccessible. We model the end-effector kinematics in simulation to study the theoretical workspace it can achieve prior to implementing a test-bench device to validate the efficacy of the end-effector. We find promising repeatability of the proposed robotic end-effector of $0.42 \mathrm{~mm}$ with an effective workspace with limits of $\pm 30^{\circ}$, which is greater than conventional neurosurgical tools. Additionally, although the tool's end-effector has a small enough diameter to operate through the narrow nasal access path and the constrained workspace of EEEA, it showcased promising structural integrity and was able to support approximately a $6 \mathrm{~N}$ load, despite a large deflection angle the limiting of which is scope of future work. These preliminary results indicate the end-effector is a promising first step towards developing appropriate handheld robotic instrumentation to drive EEEA adoption.

Keywords: Medical robotics, Neurosurgery, Robotic-Assisted Minimally Invasive Surgery, Robotic-assisted endonasal approach

\section{Introduction}

Robotic-Assisted Minimally Invasive Surgery (RAMIS) has allowed for precise and accurate instrument dexterity and articulation despite reaching anatomical sites with restricted access. ${ }^{1}$ This trait could highly benefit complex procedures such as those in the field of neurosurgery. ${ }^{2}$ Due to its delicate subject matter and challenging operations, neurosurgery has always been in need for adapting new techniques and technologies, and surgical robotics could be one such adaptation for both brain and spine applications. ${ }^{3}$

A specific neurosurgical procedure that would greatly benefit from robotic articulation in instrumentation is the Endoscopic Endonasal Transphenoidal Surgery (EETS). The EETS approach is a Minimally Invasive Surgery (MIS) technique that is performed via an anterior sphenoidotomy and aims at the removal of sellar and parasellar lesions ${ }^{4}$ with the use of an endoscope and standard rigid instru- ments. In recent years, there has been an increased interest in the Expanded Endoscopic Endonasal Approach (EEEA) that expands the EETS areas of interest to include the regions from the cribriform plate of the anterior cranial fossa to the foramen magnum in the anteroposterior plane. ${ }^{5} \mathrm{Al}-$ though a promising alternative to transcranial approaches, that require craniotomies and brain retraction, the EEEA comes with its limitations. $74 \%$ of surgeons asked in one study, ${ }^{6}$ identified the limited surgical manipulation that the standard non-articulated instruments offer as the biggest challenge of the EEEA.

In this study, we report the development of a novel design for a 3 degrees-of-freedom (DoF), tendon-driven, spherical joint robotic end-effector intended for a handheld robotic tool that aims to aid the EEEA. The end-effector is actuated by a test-bench setup that will be replaced by the handheld mechanism in future work. The design of the end-effector which is presented in this work, and a prelim- 
inary concept rendering of how the handheld robotic tool could be realised are shown in Fig. 1, alongside its potential application and workspace within EEEA. Our tendondriven mechanism has potentially increased distal-tip dexterity over standard instrumentation and can be fabricated in a small diameter profile. Our initial experiments suggest that the end-effector achieves favourable structural integrity, repeatability and workspace reach. Thus, despite the early stages of prototyping, we believe it can be fabricated such as to avoid breakage and fatigue limitations. This robustness is a result of the fact that a spherical joint can concentrate a relatively large amount of material despite being manufactured in such constrained dimensions.

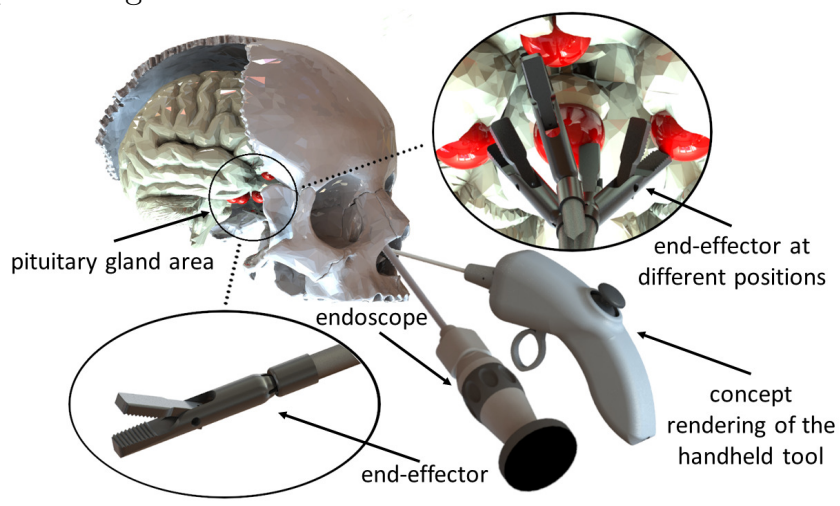

Fig. 1. Concept rendering of the handheld robotic tool and the proposed end-effector. The red spheres represent tumours on the pituitary gland (center of the brain) and besides it. The articulation of the robot can aid in the removal of tumours from these areas that often are in places where conventional tools have difficulty in reaching, or can not reach at all.

\section{Related Work}

Robotic manipulators to aid in surgery is an extensive research field which specifically for general MIS procedures, is densely populated with systems that aim to expand the capabilities of the surgeon. One such manipulator is an intraocular robotic snake intended for retinal microsurgery. ${ }^{7}$ This manipulator is actuated through a miniaturized articulated segment controlled by 4 nitinol tendons. Similar snake-like manipulators have been developed for Natural Orifice Translumenal Endoscopic Surgery (NOTES) procedures. Notable examples are an articulated distal tip with seven independently controllable DoF arranged as two universal joints, ${ }^{8}$ a robotic platform that includes a snake-like robotic endoscope equipped with a camera, a light-source and two robotic instruments, ${ }^{9}$ and the IREP (Insertable Robotic End-effectors Platform) robotic platform, a system with continuum robots with active and passive flexible segments that could also be used in different single-port access surgical procedures. ${ }^{10}$ Expanding away from these procedures, snake-like robotic manipulators have also been used to aid the treatment of osteolysis, namely a continuum dexterous manipulator with a pass-through channel that houses a debriding tool. ${ }^{11}$
Robotic platforms have also been developed for minimally invasive neurosurgery, although they appear with a lower frequency than systems for general MIS. Such robotic platforms include the Neuromate (Renishaw Mayfield, Lyon, France ${ }^{12}$ and the the Pathfinder (Prosurgics, High Wycombe, United Kingdom $)^{13}$ which are stereotactic robots used for neurological applications such as deep brain stimulation and endoscopy. Even though there are promising robotic systems intended for minimally invasive neurosurgery, the majority of commercial and research platforms still concentrate in aiding stereotactic and image-guided approaches rather than keyhole neurosurgery. ${ }^{14}$ This is mostly due to less invasive neurosurgical techniques being highly challenging, especially because of their constrained workspace. ${ }^{15}$

Specifically during EETS procedures, the workspace is exceptionally limited and constrained by the nasal access path. The surgical workspace is most closely represented by a cylinder ${ }^{16}$ with a narrow middle diameter and an average volume of $15.97 \mathrm{~cm}^{3}$, an average length of $53.9 \mathrm{~mm}$ and an average widest width of $19.08 \mathrm{~mm}$. The fact that multiple rigid manual instruments must work inside such a constrained space, makes the procedure challenging even for expert surgeons.

Motivated by this demanding technical workspace, a number of studies have reported robotic instruments for EETS, as well as robotic holders, robots assisting in the positioning and the adjustment of tools. ${ }^{17-19}$ One such robotic instrument for the EETS is a concentric tube bimanual teleoperated endonasal skull base surgery system. ${ }^{20} \mathrm{~A}$ similar platform consisting of concentric tube continuum robots has been developed ${ }^{21}$ and evaluated, ${ }^{22}$ with the authors of these studies conducting a phantom pituitary tumour removal clinical experiment. Despite some of the possible advantages of concentric tube robots, there are still concerns about the distal-end dexterity of these manipulators and their force-delivery capabilities. Taking a different robotic paradigm, a 2 DoF robotic tool with elastic elements has been introduced ${ }^{23}$ and expanded ${ }^{24}$ to a 4 DoF elastic elements robotic instrument implementing a forceps-principle. Limitations arose when the repeated deformation of the tool lead to fatigue and potential breakage.

Aiming for the same procedure, a cable-based actuation method for EETS has been reported and tested on a $5.8 \mathrm{~mm}$ DoF steerable robot, ${ }^{25}$ as well as a $5 \mathrm{~mm}$ visible forceps manipulator with a novel linkage bending mechanism, which realizes the flexible bending capability and high output force. ${ }^{26}$ While promising, these studies present instruments with larger diameters that could limit the workspace of endonasal surgery, especially when multiple tools are inserted through the same nostril. Apart from surgical manipulators, promising improvements have also been made in the field of robotic endoscopes, with one study presenting a $3 \mathrm{~mm}$ steerable endoscope with a bending angle of $65^{\circ}$ controlled by a foot switch ${ }^{27},{ }^{28}$

As previously mentioned, in order to develop a robotic end-effector that was both dexterous and robust, we decided to develop a spherical-joint end-effector. The idea of 
using a spherical joint in a miniature robotic tool has been explored and validated, ${ }^{29}$ without, however, a robotic tool implementation in this particular study. In another work, ${ }^{30}$ a spherical joint is used as part of a robotic instrument aimed for general MIS. In this work, the spherical joint is part of a larger continuum robot body rather than being the focal point of the development. As referenced in the study, a limitation of having a spherical joint for wrist dexterity as the last joint of a robotic tool, is that the instrument is compressed under the high tension required to straighten the joint, something not applicable when the spherical joint acts as the sole manipulation means.

In summary, one of the main challenges when building a robotic end-effector to aid in the EEEA, as is evident in pre-existing studies, is developing a dexterous miniature end-effector that expands the limited workspace of the current tools, while simultaneously being durable and reliable. Our suggestion to solve this problem is utilizing an existing joint-design, the spherical joint, and applying it to our endeffector by way of specific design considerations, namely the dimensions, parts-coupling and tendons placement, in such a way that would serve our purposes, since this joint type allows for dexterity while concentrating a large amount of material in limited space and thus being durable. By using the spherical joint, that has been commonly used in other works, as the focal point of our development, we have resulted in an itself novel design for a neurosurgical endeffector.

\section{Materials and Methods}

\subsection{Design and Fabrication}

When designing the robotic end-effector the main constraint we have is the very limited workspace. In transsphenoidal surgery, the main surgical workspace is the sphenoidal sinus which is mostly resembled by a $25 \mathrm{~mm}$ diameter sphere. ${ }^{31}$ This limitation, combined with the narrow passage that the tool must follow to reach the sinus and the fact that more than one instruments are often used through the same nostril at the same time, lead to the need for a small diameter. Our proposed robot mechanism has a diameter of $3.6 \mathrm{~mm}$ and a length of $19.4 \mathrm{~mm}$. Commercially available tools for endonasal surgery have diameters in the range of $1 \mathrm{~mm}$ to $7 \mathrm{~mm}$, and end-effector lengths in the range of approximately $10 \mathrm{~mm}$ to $20 \mathrm{~mm},{ }^{32}$ which deem the proposed end-effector adequately small and simultaneously big enough to avoid breakages as discussed in Section 4.2 .

We base the end-effector's articulation properties around the use of a spherical joint design rather than a more traditional articulated design. This is motivated by using the spherical joint as a platform for easy tool adaptation and also for potentially enhanced robustness since a sphere concentrates a large amount of material and is difficult to break. Six nylon tendons, 2 for each DoF, with a diameter of $0.25 \mathrm{~mm}$ terminate on the distal end of the sphere, passing through $0.5 \mathrm{~mm}$ diameter channels. The end-effector of the robot is coupled on the distal end of the sphere completing the robot assembly.

Additive manufacturing, and more specifically stereolithography (SLA), was used to print the initial implementation of our $3.6 \mathrm{~mm}$ robotic end-effector. The printing material was clear resin, a type of resin used in fluidics, optics and mold-making. This material was chosen because it allows for printing resolutions as small as $25 \mu \mathrm{m}$. The printer used for this manufacturing was the desktop 3D printer Form 2 (FormLabs, Somerville, Massachusetts, United States).

The robot end-effector is fixed on a stainless steel grade 316 seamless tube shaft of $3 \mathrm{~mm}$ outer diameter and all the tendons pass through $0.457 \mathrm{~mm}$ diameter 304 welded hard drawn stainless steel wire-guiding tubes, that do not allow the tendons to get tangled with each other. This $3 \mathrm{~mm}$ shaft is then fixed on a test-bench setup that was used to validate the end-effector's efficacy. The test-bench setup consists of an upper tendon-housing part that contains the axes where the tendons terminate and their rerouting system, and a lower actuation part with all the motors. It was again produced via additive manufacturing techniques, this time in polylactic acid (PLA) plastic using the desktop 3D printer Ultimaker S5 (Ultimaker, Geldermalsen, Netherlands).

The tendons starting from the joints of the end-effector terminate on capstans that lie on three $3 \mathrm{~mm}$ diameter rigid stainless steel shafts on the tendon-housing part, which are co-axially connected with the motor shafts on the actuation part of the system. Each DoF is controlled by two nylon tendons that are fixed on capstans on the same rigid shaft. Since the tendon insertion points from the shaft to the test-bench upper part are not colinear with their corresponding capstans, there is also a rerouting mechanism that uses pulleys to redirect the tendons from their one point to the other in order to reduce their backlash and friction. The rerouting mechanism as well as the tendons insertion points are shown in Fig. 2.

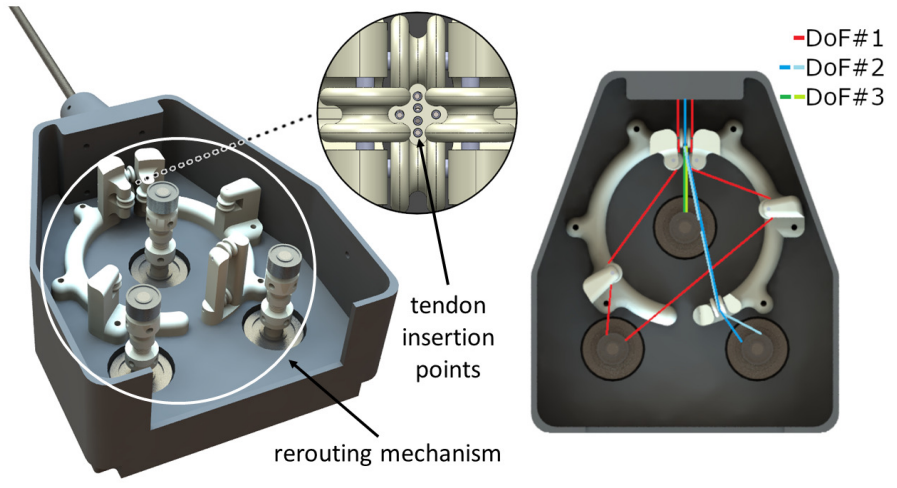

Fig. 2. CAD designs of the rerouting mechanism with the tendon insertion points (left), and the tendon rerouting paths (right).

For a deeper understanding of how the tendon pulling 
and pushing leads to robot joint movement, Fig. 3 depicts the physical relationship between the tendons of one of the robot DoF and the corresponding robot joint.

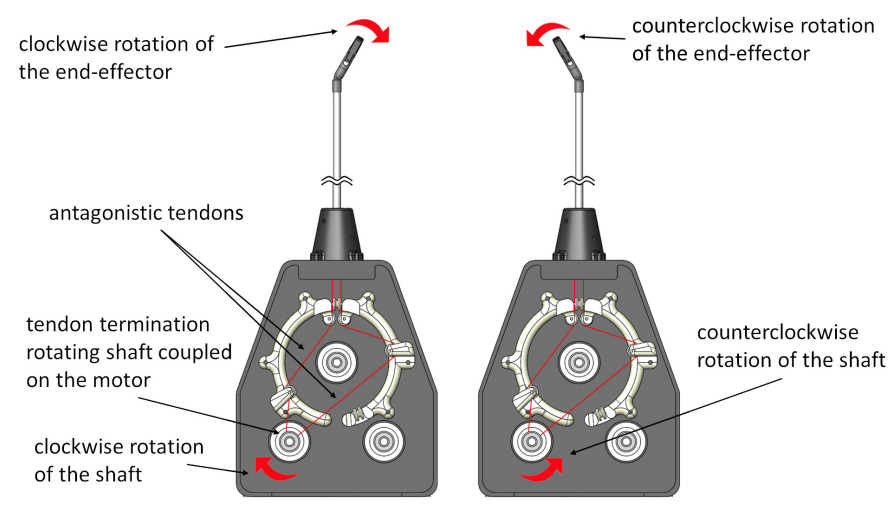

Fig. 3. Physical relationship between one of the robot joints and its corresponding tendons.

The motors used for the actuation system were three MX-28T Dynamixel Robot Servo Actuators (Robotis CO., Ltd, South Korea) with a stall torque of $25.5 \mathrm{~kg} \cdot \mathrm{cm}$ and an angular step resolution of $0.088^{\circ}$. Each actuator utilizes an embedded PID-controller and communicates through a USB-to-TTL bus at a rate of $50 \mathrm{~Hz}$. Motor selection for this setup was based on the torque capabilities of the motors and their seamless integration into the system, a result of their embedded encoders and controllers. A more sophisticated selection of miniature motors will need to be considered during the development of the handheld tool. The motors are fixed on a PLA-prototyped base and covered by a PLA mount-plate while on the rotating shaft of each motor an SLA-prototyped coupler is fixed. The mount-plate of the actuation part of the test-bench is equipped with a coupling pattern on which the tendon-housing part is fitted, while the motor shaft couplers connect with the rigid rotation shafts that stand inside the upper part. In this way, the actuation system is entirely separated from the tendon-housing which allows for easy swapping and testing of the different end-effectors that have been prototyped.

A complete overview of the test-bench system and the end-effector, both in CAD and its actual implementation, is shown in Fig. 4.

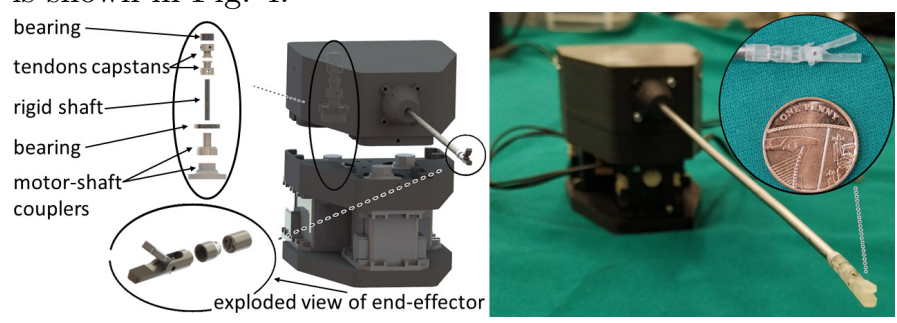

Fig. 4. (Left) CAD renderings of the test-bench system and the end-effector. The top-left circled assembly indicates the $3 \mathrm{~mm}$ diameter rigid stainless steel shaft on which the tendons terminate, whereas the top-down circled assembly is an exploded view of the end-effector. (Right) Implementation of the testbench system and the end-effector.

\subsection{Kinematic analysis}

\subsubsection{Forward Kinematics}

Although a spherical joint design was used, the joint is rotating over two axes only, namely the pitch and yaw axes, and not over the roll axis since the tendons and antagonistic motion constrain such rotation. The missing roll motion, as well as the translation of the tool, will be carried out by the surgeon's hand when the handheld robotic tool is implemented in future work. The kinematic model of the robot is extracted using the Denavit-Hartenberg (DH) convention with the frames of the first two DoF located on the middle point at the base of the spherical joint, while the frame of the third DoF is located on the axis of rotation of the gripper end-effector. The end-effector frame is located on the distal middle point of the robot, namely on the bottommiddle part of the rotating part of the gripper. The joint frames of the robotic end-effector are shown in Fig. 5, and the produced DH parameters are found in Table 1.

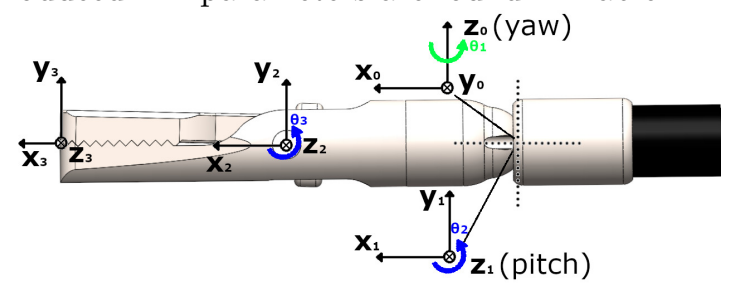

Fig. 5. Robotic end-effector joint frames alongside the $\left[\theta_{1}, \theta_{2}, \theta_{3}\right]$ angles.

Table 1. The DH parameters of the $3.6 \mathrm{~mm}$ robotic end-effector.

\begin{tabular}{|c|c|c|c|c|}
\hline $\boldsymbol{i}$ & $\boldsymbol{a}_{\boldsymbol{i}}(\boldsymbol{m m})$ & $\boldsymbol{\alpha}_{\boldsymbol{i}}(\boldsymbol{r a d})$ & $\boldsymbol{d}_{\boldsymbol{i}}(\boldsymbol{m m})$ & $\boldsymbol{\theta}_{\boldsymbol{i}}(\boldsymbol{r a d})$ \\
\hline $\mathbf{1}$ & 0 & $\pi / 2$ & 0 & $\theta_{1}$ \\
\hline $\mathbf{2}$ & 9.6 & 0 & 0 & $\theta_{2}$ \\
\hline $\mathbf{3}$ & 9.8 & 0 & 0 & $\theta_{3}$ \\
\hline
\end{tabular}

\subsubsection{Workspace Analysis}

In Fig. 6, we compare the target-reaching capabilities of the proposed end-effector to that of traditional tools using the open-source software CHAI3D. ${ }^{33}$ This simulator was implemented using the robot's forward kinematics equations and was controlled by a 6 -DoF joystick that controls the robot joints and the shaft movement in 3D space. Alongside the robotic end-effector, a traditional non-articulated tool was implemented in the same framework, again controlled by the joystick. The aim of this simulator was to test the robot kinematics and showcase that some areas besides 
the pituitary gland and around the base of the brain can be reached with the robotic end-effector but are out of reach with the conventional tool. This is depicted when all three red points can be reached and turn to green with the articulated tool, but only one of them with the traditional non-articulated end-effector.

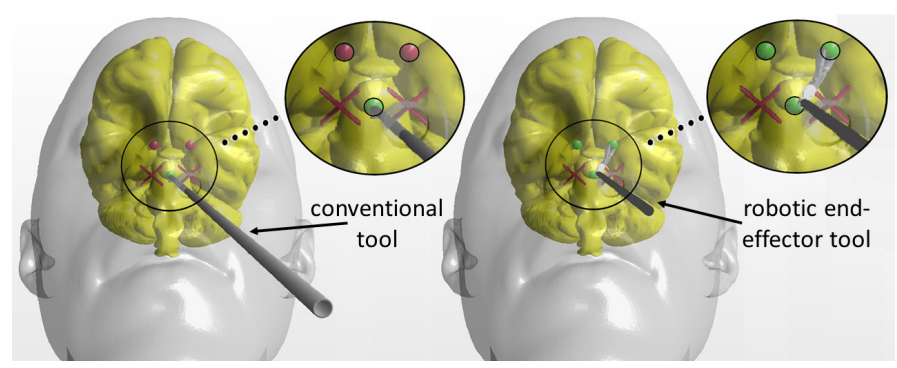

Fig. 6. Reaching areas beneath the pituitary gland with a simulated traditional instrument (left), and with the proposed robotic end-effector (right). Red points represent points the conventional tool can not reach whereas the green points represent the points the tools can reach. Red Xs represent no-go areas besides the pituitary gland.

Expanding on this preliminary proof-of-concept workspace analysis of the end-effector, a more thorough analysis was carried out in the robot simulation platform CoppeliaSim. ${ }^{34}$ Both the robotic end-effector and a nonarticulated tool with the same dimensions were simulated in this environment. To also simulate the physical constraint within which the tools operate during surgery, a 3D model of a cranial CT scan, modified so that it only features the EEEA areas of interest, was also input into the simulation platform. Apart from the cropping of the 3D model to exclude areas of the skull that are out of scope for this procedure and would slow down the simulation, a cylindrical channel was also created starting at the sphenoid ostium, and passing through the sphenoid sinus granting access to the pituitary gland area. This channel is created by the surgeon during the EEEA procedure to access the pituitary gland, and is highly constraining the tool motion. The diameter chosen for the the cylindrical channel is $1 \mathrm{~cm} .{ }^{4}$ The modified 3D model is shown in Fig. 7 .

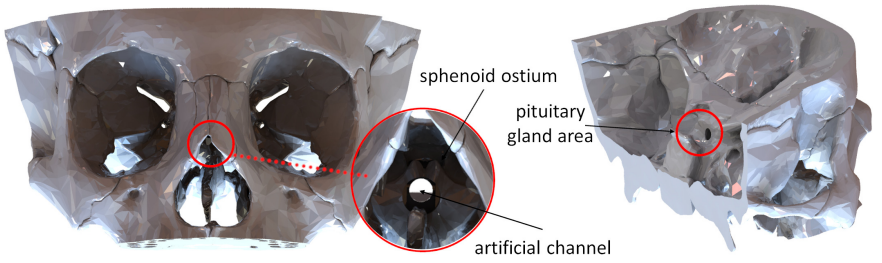

Fig. 7. 3D model of a cranial CT scan used to determine the available workspaces during EEEA. The artificial channel shown inside the circled areas represents the channel that the surgeon creates during surgery and grants access to the pituitary gland area.
The goal of this workspace analysis was to compare the effective workspace of the non-articulated tool with that of the suggested robotic end-effector. To calculate both workspaces, a search was carried out that iterated through each tool's various DoF. The non-articulated tool was simulated by a 5 DoF system. 3 DoF for the $(x, y, z)$ translation of the shaft, and 2 DoF for the (yaw, pitch) rotation of the shaft. The robotic end-effector was modelled similarly, with the addition of $2 \mathrm{DoF}$ for the end-effector joints. In both cases the roll DoF, as well as the gripper open and close DoF, were not taken into consideration for simplification reasons. The DoF coordinate frames for both tools on a side-by-side comparison can be seen in Fig. 8 .

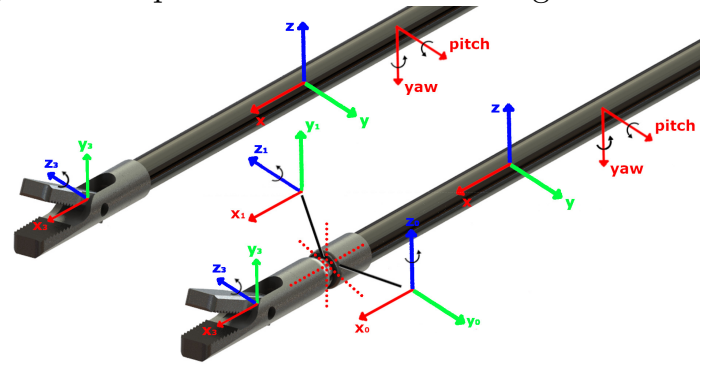

Fig. 8. DoF coordinate frames of the traditional nonarticulated tool (left), and of the suggested robotic end-effector (right). The robotic end-effector comprises of an additional two articulated joints represented by frames $\left(x_{0}, y_{0}, z_{0}\right)$ and $\left(x_{1}, y_{1}, z_{1}\right)$.

The workspaces were evaluated by accessing a number of different shaft positions, entering from the right side of the nasal cavity, and different shaft and robot-joints orientations. The range of translation of the shaft was $20 \mathrm{~mm}$ with an increment of $0.6 \mathrm{~mm}$ on each axis, the range of rotation of the shaft was $10^{\circ}$ with an increment of $0.5^{\circ}$ on each axis, and the range of the robotic end-effector DoF joint-space angle vector was $70^{\circ}$, from $-35^{\circ}$ to $35^{\circ}$, discretised by $5^{\circ}$. These specific ranges and steps were a result of trial-and-error testing to find the most suitable values. The physical constraint was incorporated by determining mesh collision between the tools and the cranial model. An endeffector point was pushed into the point cloud only if no part of the tool was in contact with the physical constraint by employing the mesh collision checking capability of the CoppeliaSim software. The resulting effective workspaces are shown in Fig. 9.

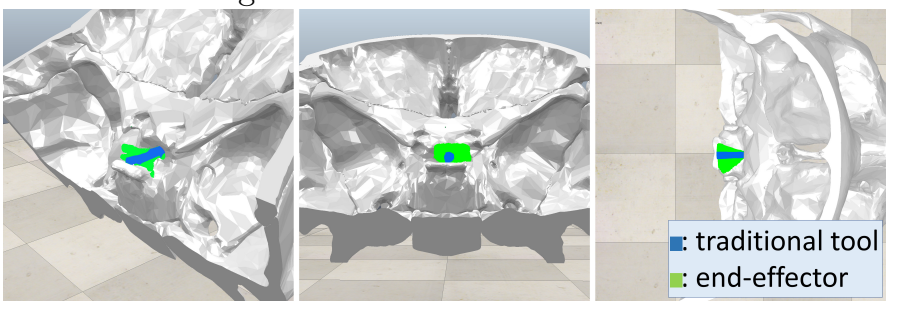

Fig. 9. Workspace comparison between the proposed robotic end-effector (green) and a traditional, non-articulated tool with the same dimensions (blue), from three different viewing angles. 
It is evident that the robotic end-effector has a broader workspace and can reach a significantly larger amount of points in 3D space, suggesting that it can potentially improve the efficacy of EEEA. Adding to this analysis, it could be useful to conduct the workspace search in the entirety of the available workspace, rather than just after the sphenoid sinus. This would require a high accuracy 3D model, inclusive of soft-tissue and other structures and could be the scope of future work.

\subsubsection{Inverse Kinematics and Tendon Lengths}

For the inverse kinematics robot model, the forward kinematics equations extracted using the Denavit-Hartenberg convention were used, resulting into closed form solutions for the calculation of $\left[\theta_{1}, \theta_{2}, \theta_{3}\right]$ as shown in Eq. 1 .

$$
\theta_{i}= \begin{cases}\arcsin \left(r_{13}\right), & \text { for } i=1 \\ \pm \arccos \left(\frac{y-0.0098 \cdot \sin \left(\theta_{1}\right) \cdot r_{32}}{0.0096 \cdot \sin \left(\theta_{1}\right)}\right), & \text { for } i=2 \\ \arcsin \left(\frac{r_{31}-\frac{\sin \left(\theta_{2}\right)}{\cos \left(\theta_{2}\right)} \cdot r_{32}}{\cos \left(\theta_{2}\right)+\frac{\sin \left(\theta_{2}\right)}{\cos \left(\theta_{2}\right)} \cdot \sin \left(\theta_{2}\right)}\right) & \text { for } i=3 .\end{cases}
$$

with the Homogeneous Transformation being: $H\left(\theta_{1}, \theta_{2}, \theta_{3}\right)=\left[\begin{array}{cccc}r_{11} & r_{12} & r_{13} & x \\ r_{21} & r_{22} & r_{23} & y \\ r_{31} & r_{32} & r_{33} & z \\ 0 & 0 & 0 & 1\end{array}\right]$.

Assuming a computed joint-space angle vector, the stroke of each antagonistic tendon pair must be computed in order to control the robot. The method used is similar to the one used in some of the authors' previous work. ${ }^{35}$ For each rotational DoF there are two tendons that control the movement of each angle in an agonist-antagonist fashion. We define as the length of the tendon the distance between the base of the robot, rather than the starting point of the tendon on the tool-housing capstan, to the point that each tendon terminates.

The lengths of the tendons are computed by locating the $3 D$ positions of the pass-through channels for every tendon and calculating the distance between two consecutive channels. As is evident in Fig. 10, the robot has six channels that tendons pass through.

Four of them, channels $i=1$ to 4 , are diametrically positioned at an $1 \mathrm{~mm}$ radius from the centre, terminate on the distal end of the sphere and control the two first DoF. The remaining two, channels $i=5$ and 6 , control the gripper end-effector DoF, are located with a $\pm 0.35 \mathrm{~mm}$ offset from the centre on the z-axis, and terminate on the gripper attachments. On the base of the robot the six holes are positioned as shown in Eq. 2 for $r_{1}=1 \mathrm{~mm}$ and $r_{2}=0.35 \mathrm{~mm}$.

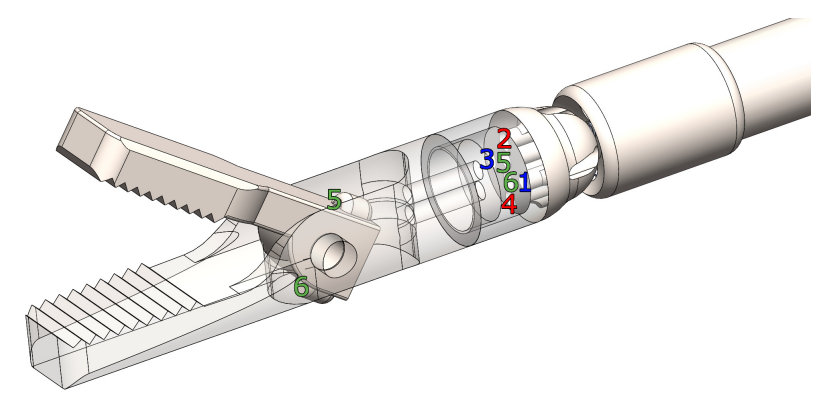

Fig. 10. The 6 channels on the spherical joint where the tendons pass through. The exact same pattern is followed on the base of the end-effector, whereas only tendons 5 and 6 arrive at the upper part of the gripper.

$$
P_{i}(x, y, z)=\left\{\begin{array}{l}
\left(x_{0}, y_{0}+r_{1}, z_{0}\right), \text { if } i=1 \\
\left(x_{0}, y_{0}, z_{0}+r_{1}\right), \text { if } i=2, \\
\left(x_{0}, y_{0}-r_{1}, z_{0}\right), \text { if } i=3 \\
\left(x_{0}, y_{0}, z_{0}-r_{1}\right), \text { if } i=4 \\
\left(x_{0}, y_{0}, z_{0}+r_{2}\right), \text { if } i=5, \\
\left(x_{0}, y_{0}, z_{0}-r_{2}\right), \text { if } i=6 .
\end{array}\right.
$$

After the channels have been positioned in $3 \mathrm{D}$ space using the forward kinematics, the resulting homogeneous transformation matrices are used to calculate the Euclidean geometric distances. Finally, the difference between the lengths of the tendon on the current joint-space and the desired joint-space is turned into the angle by which the motor should rotate in position control.

The positions of the channels $P_{i}{ }^{\prime}$ and $P_{i}{ }^{\prime \prime}$, for channel $i=1$ to 6 , in $3 \mathrm{D}$ space for a specific joint-space angle vector $\left[\theta_{1}, \theta_{2}, \theta_{3}\right]$ are calculated using Eq. 3 and Eq. 4. $P_{i}{ }^{\prime}$, for channels $i=1$ to 4 , represents the positions where the corresponding tendons terminate, whereas for channels $i=5$ and $i=6, P_{i}{ }^{\prime}$ represents the passing-through channels on the spherical joint for tendons 5 and 6 . The termination points of the latter pair of tendons is represented by the $P_{i}{ }^{\prime \prime}$ position pair.

$$
\begin{aligned}
P_{i}{ }^{\prime}(x, y, z) & =A_{0 i}^{1} \cdot P_{i}(x, y, z), \text { for } i=1 \text { to } 6, \\
P_{i}{ }^{\prime \prime}(x, y, z) & =A_{0 i}^{2} \cdot P_{i}(x, y, z), \text { for } i=5 \text { and } 6,
\end{aligned}
$$

where $A_{0 i}^{j}$ is the Homogeneous transformation that corresponds to joint $j$ and channel $i$. Then, the Euclidean distance $L_{i}$ between two consecutive channels is:

$$
\begin{aligned}
L_{i}= & \left\|P_{i}{ }^{\prime}(x, y, z)-P_{i}(x, y, z)\right\|, \text { for } i=1 \text { to } 4, \\
L_{i}= & \left\|P_{i}{ }^{\prime}(x, y, z)-P_{i}(x, y, z)\right\|+ \\
& \left\|P_{i}{ }^{\prime \prime}(x, y, z)-P_{i}{ }^{\prime}(x, y, z)\right\|, \text { for } i=5 \text { and } 6,
\end{aligned}
$$

and to compute the displacement of tendon $i$ between two joint-space angle vectors $j$ and $j^{\prime}$, the tendon lengths $L_{i}^{j}$ and $L_{i}^{j^{\prime}}$ need to be computed and subtracted. 


$$
\Delta L=\left|L_{i}^{j}-L_{i}^{j^{\prime}}\right|
$$

A visual example of how these lengths are computed for tendon 2 is shown in Fig. 11.

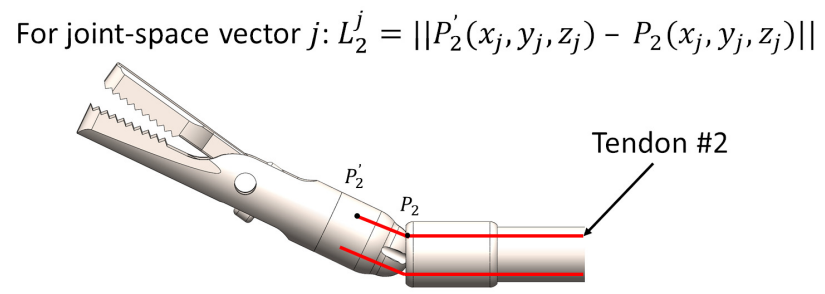

For joint-space vector $\mathrm{j}^{\prime}: L_{2}^{j^{\prime}}=\left\|P_{2}^{\prime}\left(x_{j^{\prime}}, y_{j^{\prime}}, z_{j^{\prime}}\right)-P_{2}\left(x_{j^{\prime}}, y_{j^{\prime}}, z_{j^{\prime}}\right)\right\|$

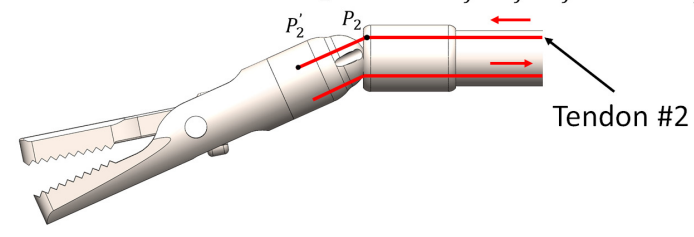

Fig. 11. Visual representation of how the length of tendon 2 is computed for two different joint-spaces $j$ and $j^{\prime}$.

Finally, to compute the angle $\phi$ by which the motor needs to rotate so that the tendons are pulled/pushed by the appropriate amount, Eq. 8 is used:

$$
\phi=\frac{\Delta L}{R_{c}},
$$

where $R_{c}$ is the radius of the capstan on which the tendon terminates. In this case capstans of radius $1.5 \mathrm{~mm}$ were used, and thus $R_{c}=1.5 \mathrm{~mm}$. Since $\Delta L$ is always positive, the sign of the angle is decided by a software convention taking into account the direction of joint movement. This joint movement direction is easy to calculate, because both the current joint-space angle vector and the target jointspace angle vector are known. The sign of each motor angle is determined by the sign of the difference between the angle of its corresponding joint at its current joint-space and the angle of its corresponding joint at the goal joint-space.

It is worth mentioning that some simplifications took place during this mathematical process. Each pair of tendons does not always move by the exact same distance because for a spherical joint, movement of DoF 1 results in minor movement of DoF 2 and vice versa. Thus, there is chance that for some joint-space angle vectors one tendon is pulled or pushed more than its antagonist is pushed or pulled. This coupling could potentially vanish by better constraining the spherical joint inside its base, or by placing the tendon channels on different positions on the joint. This difference in tendon lengths between antagonistic pairs, however, and more specifically between tendon pairs 1 and 3,2 and 4 , and 5 and 6 , we found out is very small numerically. Since we have one motor per tendon pair, we decided to disregard this difference and base the rotation of the motor on the average of the two lengths, without trying to build a better base constraint for the spherical joint. Another simplification is that in this analysis we have not taken into account tendon bending radii that would appear in extreme joint angles because of the geometry of the spherical joint.

\subsection{Experimental Methods}

\subsubsection{Workspace and Repeatability Study}

The robotic end-effector was tested for its repeatability and workspace capabilities. The focus was on repeatability rather than accuracy since in RAMIS procedures the robot is either tele-operated via a master console or directly controlled with a handheld mechanism by the surgeon making sure the desired motions are achieved under endoscopic view. For both tests, optical tracking was employed to measure the position of the end-effector. More specifically, two optical markers that were fixed on an SLA-printed attachment were tracked in 3D space. The attachment was again printed in clear resin using the Form 2 desktop 3D printer. The end-effector was firmly gripping the marker attachment so that the tracked positions of the markers could be used to extract the position of the end-effector as detailed later in the section.

For the repeatability test, a vector of 19 desired workspace joint values uniformly generated within the joint-space range between $[-30,-30,30]$ and $[30,30,30]$ was created, with $\theta_{3}=30$ the gripper angle required to get a good grip on the SLA-printed tracking marker attachment. The robotic end-effector was instructed to move between those 19 points, and each time a movement was finished the positions of the markers in $3 D$ space were recorded with the NDI Optotrak Certus motion capture system (Northern Digital Inc, Canada).This system has a tracking accuracy of $0.1 \mathrm{~mm}$ and a resolution of $0.01 \mathrm{~mm}$. The robotic end-effector and its test-bench were positioned in front of the tracking system so that the markers are in direct line-of-sight of its three cameras.

Both the robot with its test-bench actuation system, and the capture system were operating within the Robot Operating System (ROS) framework. ${ }^{36}$ The application consisted of two nodes. Within the first node the kinematic computations took place and the motors were instructed to rotate by a specific angle, which corresponded to the desired end-effector position, as shown in Section 3.2. To ensure that the motors had reached their desired position before moving on to measure the position of the tracking markers, a 'do-while' logic was implemented. The desired position was fed into the motors until the current position was measured to be within a threshold of $0.18^{\circ}$ maximum error in motor angle. The capture system was operating within the second node and was constantly publishing transformations that the first node would look up every time it was instructed. When these transformations 
were obtained, they would be stored in a rosbag file. The data flow between these two nodes is shown in the flow diagram of Fig.12.

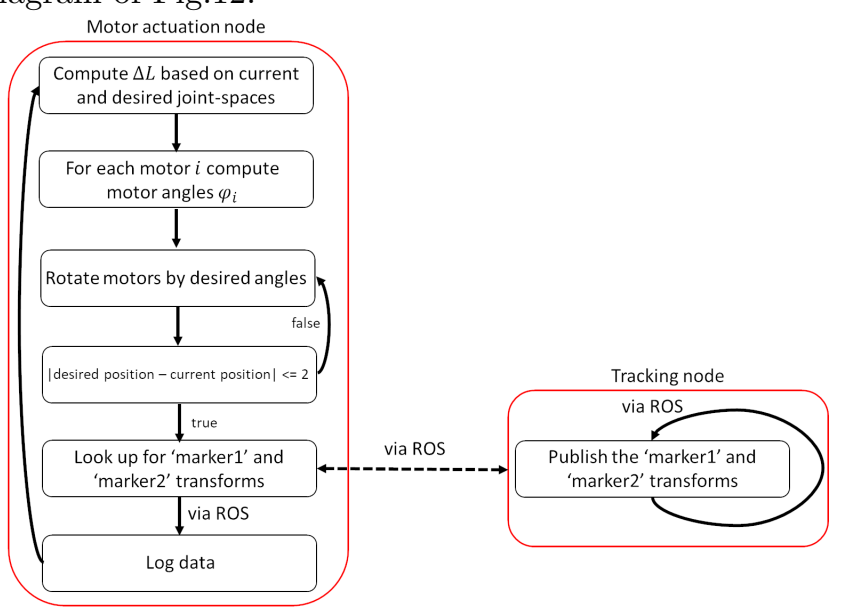

Fig. 12. Flow diagram showing the interaction between the two ROS nodes of the tracking experiment.

The robot end-effector gripping the marker attachment with the NDI markers on it can be seen in Fig. 13. In the same figure, and for demonstration purposes, the end-effector inside a training head phantom is shown. This picture provides an illustration of the robotic EEEA, while simultaneously relating the dimensions of the instrument shaft to those of the phantom head.
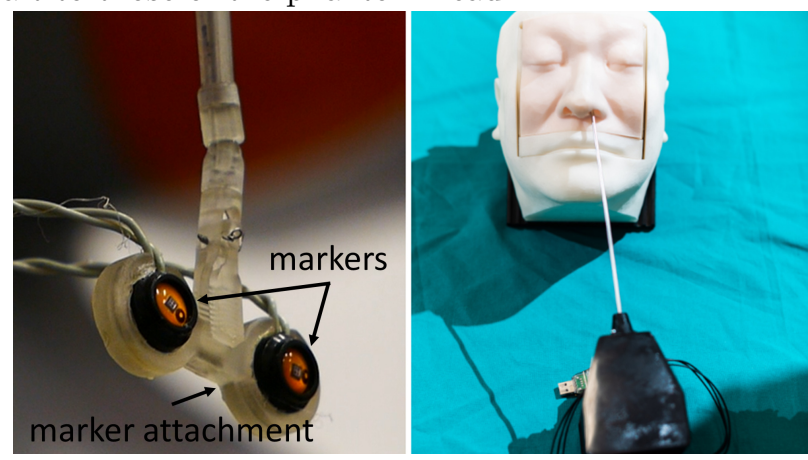

Fig. 13. The robotic end-effector holding the NDI tracking markers attachment (left), and inside a training phantom to illustrate insertion during the robotic EEEA procedure (right).

The SLA-printed marker attachment was designed in a way so that the line connecting the centres of the two markers passes by the distal point of the gripper, where the last frame of the end-effector was mathematically defined. Thus, the robot end-effector position could be represented by the mean point between the two marker-point positions. Since we are only concerned with position and not orientation, just the two markers are sufficient for our purposes. The markers where tight-fitted and hot-glued to the attachment to avoid shifting or falling out of place, and a number of different gripper angle values were tried mak- ing sure that the gripper holds on the attachment tightly enough. This was essential to make sure that the measurements kept were indicative of the robot movements.

After all 19 moves were carried out, the vector of points was shuffled and the robot was instructed to rotate by the same joint-space values, but in a different order. By shuffling the desired joint-space values we reduce the bias in the measurements that following the same order of values every time would produce. The vector of values was shuffled 19 times, resulting in 20 different sequence groups and 380 moves overall.

To test the workspace capabilities of the robot, it was instructed to move to the boundaries of the joint-space, in the range between $[-30,-30,30]$ and $[30,30,30]$. After the robot reached every position, again the positions of the tracking markers in 3D space were recorded to be used for the construction of the robot end-effector workspace boundaries. Finally, to confirm that the robot is rotating around fixed axes, the end-effector was instructed to move into 1000 random joint values between the same range $[-30,-30,30]$ and $[30,30,30]$. The resulting positions were tracked using the NDI trackers and then organised into a point cloud. The fitted sphere on the workspace point cloud was calculated with the purpose to measure its radius and compare it to the distance between the first and the last frame of the robot end-effector as shown in Fig. 5.

\subsubsection{Structural Integrity study}

Considering its miniature size, it was important to test the structural integrity of the end-effector and the amount of load it can support without its gripper or any other part of the robot breaking. To test that, a set of precision weights in the range of $100 \mathrm{~g}$ to $700 \mathrm{~g}$ was tied on an SLA-printed attachment using stainless steel wire. Then, a two-pulley system was used to transfer the weight of each object along the wire and change its direction so that the end-effector can grasp the attachment and support the object. The pulleys were of the same size, were placed at the same height and the wire did not wrap around the pulleys, only passed above them. Disregarding the losses generated by the friction on each pulley, it is acceptable to assume that the effort required by the robot to support the object is almost equal to the weight of the object. The experimental setup for the end-effector's structural integrity is shown in Fig. 14.

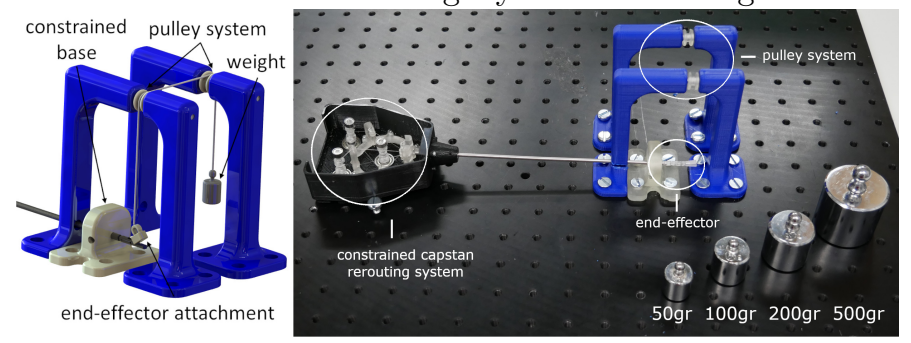

Fig. 14. Rendering (left), and implementation (right) of the experimental setup to test the end-effector's structural integrity. 
The part of the rerouting system that controls the first two DoF tied to the spherical joint movement was constrained by tying an extra tendon around the capstans that disallowed them to move. This was done in order to make sure that any deflection appearing on the axis of the spherical joint was solely due to the effort created by the weight, rather than a normal rotation of the joint. The only DoF that was free to move was that of the gripper. As is evident in Fig. 14 the tool-housing is unattached from the actuation system and the gripper was moved manually rather than using its designated motor. This approach was chosen to simplify the experiment design and since the purpose of the experiment is to test the mechanical integrity of the end-effector, the motors are not necessary.

One by one the objects were tied to the attachment and each time the end-effector was tasked with the grasping of that attachment. The weight attachment was placed on the top part of the gripper of the end-effector with the weight left to freely hang from the pulley-system. The rigid shaft on which the gripper tendons terminate was then manually rotated until we saw the gripper closing and the weight being lifted. Depending on the weight, a deflection angle appeared. Here, the deflection angle is defined as the angle formed on the pitch axis of the spherical joint between the resting position and the final position of the end-effector. This angle is later illustrated as part of the experimental results in Fig. 16. The end-effector was evaluated on its abilities to maintain a grasp on the attachment and support the weight for $15 \mathrm{sec}$. For each object, the deflection angle was measured. One prototype was used throughout this series of experiments and each weight was tested twice. Between the two series of experiments, the difference in deflection angles for the same weight were negligible.

\section{Results}

\subsection{Workspace and Repeatability}

To calculate the repeatability value of the robot endeffector, all 19 tracker transformation measurements that correspond to the same joint-space values from each of the 20 iterations are put into a group and their standard deviation (STD) is calculated, resulting in 19 different STD values. Each STD value represents the repeatability of that specific joint-space value and physically corresponds to the positional deviation from the average of displacement. ${ }^{37}$ These values rounded up on the second decimal digit, and the joint-space they correspond to, can be seen in Table 2 . We define as the overall repeatability of the robotic endeffector, the average of those STD values. Using this procedure, it was found that the repeatability of the robot is measured at $0.42 \mathrm{~mm}$.

Table 2. The STD values of the 19 different joint positions.

\begin{tabular}{|c|c|c|c|}
\hline $\left.\boldsymbol{J o i n t}_{(}{ }^{\boldsymbol{o}},{ }^{\boldsymbol{o}}\right)$ & $\boldsymbol{S T D}(\mathbf{m m})$ & $\left.\boldsymbol{J o i n t}^{(\boldsymbol{o}},{ }^{\boldsymbol{o}}\right)$ & $\boldsymbol{S T D}(\mathbf{m m})$ \\
\hline$(-21,-29)$ & 0.29 & $(-19,-23)$ & 0.43 \\
\hline$(18,-27)$ & 0.21 & $(15,-21)$ & 0.39 \\
\hline$(4,14)$ & 1.02 & $(-9,5)$ & 0.39 \\
\hline$(-12,-1)$ & 0.48 & $(15,-1)$ & 0.26 \\
\hline$(15,-30)$ & 0.20 & $(-13,4)$ & 0.33 \\
\hline$(6,23)$ & 0.12 & $(-5-22)$ & 0.32 \\
\hline$(-25,16)$ & 0.82 & $(-12,-22)$ & 0.23 \\
\hline$(-12,-12)$ & 0.66 & $(24,18)$ & 0.94 \\
\hline$(11,-19)$ & 0.39 & $(-19,29)$ & 0.26 \\
\hline$(7,-27)$ & 0.19 & & \\
\hline
\end{tabular}

The boundaries of the workspace of the robot end-effector, as recorded using the NDI tracking markers, compared to the corresponding theoretical boundaries for the joint-space range of $[-30,-30,0]$ to $[30,30,0]$ are shown in Fig. 15 .
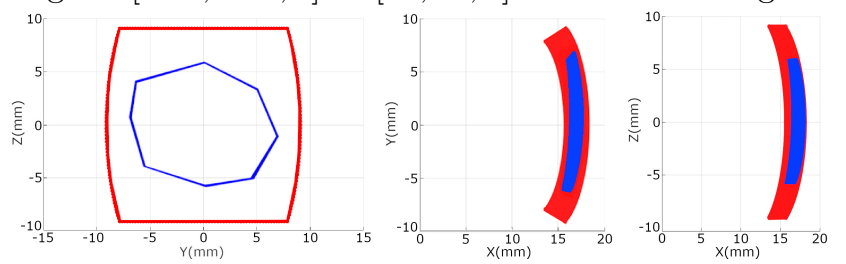

Fig. 15. Comparison between the boundaries of the theoretical workspace (red) and the measured workspace (blue).

According to these tracking data, the maximum distance that the robot end-effector can cover while constrained to a fixed point, which is the origin of frames 0 and 1 as shown in Fig. 5, is $13.1 \mathrm{~mm}$ on the Y-axis, whereas on the $\mathrm{Z}$-axis is $12.5 \mathrm{~mm}$. The theoretical value for both these axes is $18.5 \mathrm{~mm}$ which means there is an error in movement range of $5.4 \mathrm{~mm}$ on the $\mathrm{Y}$-axis and another one of $6 \mathrm{~mm}$ on the Z-axis. It is worth mentioning that although the endeffector has a fixed point where the axes of rotation pass through, its purpose is to be integrated into a handheld mechanism and held by the surgeon that would be moving that fixed point in space, allowing thus for a wider and more sophisticated effective workspace. This is why it was important to verify whether this point was in fact fixed or whether any slides in movement were present that could lead to inaccuracies in the measurements.

As previously mentioned, to confirm that the robot is indeed rotating around fixed axes, we calculated the fitted sphere on the point cloud created by moving the endeffector to 1000 random joint values between the previously specified joint-space angle vectors. The resulting sphere has 
a radius of $19.6 \mathrm{~mm}$ and a fitting error of $29.1 \mu \mathrm{m}$.

The actual distance between the axis of rotation and the distal point of the robot end-effector, the movement of which was tracked, was measured at $19.4 \mathrm{~mm}$ as also mentioned in Section 3.1, using vernier calipers with a resolution of $0.01 \mathrm{~mm}$, comparable to the fitted sphere radius, verifying that the rotating axis of the robot is not sliding. The tendons are responsible for keeping the base of the robot end-effector fixed to the distal end of the instrument shaft through tension. The robot end-effector is not otherwise fixed to the shaft.

\subsection{Structural Integrity}

The end-effector supporting the objects and the corresponding deflection are shown in Fig. 16. The end-effector was able to support all the objects up to a mass of $600 \mathrm{~g}$, which corresponds to approximately $6 N$, and was able to maintain a grasp up to a mass of $500 \mathrm{~g}$. Here, a successful grasp is defined by the upper part of the gripper not moving from its 'grasping' position for the whole duration of the $15 \mathrm{sec}$, after the rigid shaft had been rotated. Thus, even though the gripper is not fully closed for some of the weights because the rigid shaft was not manually rotated to its full extent, as is evident in Fig. 16, the grasping test for these corresponding weights was still considered successful.

For the $600 \mathrm{~g}$ object it could not maintain a grasp and the gripper was gradually opening as the seconds passed by, even though the capstans and the shaft connected to the gripper-tendons were manually kept still. For the $700 \mathrm{~g}$ object, the gripper was able to support the weight for barely $3 \mathrm{sec}$ before opening and letting go of it. The results of this structural integrity study are concentrated in Table 3.

Table 3. The results of the structural integrity study.

\begin{tabular}{|c|c|c|c|}
\hline Effort $(\sim \boldsymbol{N})$ & Deflection $\left.^{(\boldsymbol{o}}\right)$ & Support & Grasp \\
\hline $\mathbf{1}$ & 15 & $\checkmark$ & $\checkmark$ \\
\hline $\mathbf{2}$ & 25 & $\checkmark$ & $\checkmark$ \\
\hline $\mathbf{5}$ & 35 & $\checkmark$ & $\checkmark$ \\
\hline $\mathbf{6}$ & 35 & $\checkmark$ & $\times$ \\
\hline $\mathbf{7}$ & 55 & $\times$ & $\times$ \\
\hline
\end{tabular}

The deflection angle presents a fairly expected behavior. It is relatively small for the $100 \mathrm{~g}$ object, bigger for the $200 \mathrm{~g}$ and for the $500 \mathrm{~g}$ and $600 \mathrm{~g}$ it reaches and even surpasses the boundaries of the effective workspace as they were calculated in the previous set of experiments. For the $700 \mathrm{~g}$ object the deflection angle is well over the effective workspace boundaries and although the end-effector did not break, such a large effort during an operation would significantly weaken the tool. By design, the forces exerted during the transsphenoidal approach are rarely larger than $2 N,{ }^{38}$ on the axis in question in our study.

\section{Discussion}

In this study, we proposed an articulated 3 DoF robotic end-effector for a novel handheld robotic tool intended for the EEEA. Using an actuation test-bench, we evaluated the design prototype in terms of its repeatability and workspace capabilities, as well as its structural integrity. The choice of a tendon-driven mechanism was based on the improved distal point abilities that such designs offer as well as their structural integrity. To be able to operate inside a very limited workspace, the robot must have a small diameter which often leads to breakages and fatigue. The proposed robot end-effector has a spherical joint in order to create a robust structure by taking advantage of the fact that a sphere can concentrate a large amount of material despite being fabricated in a small size.

During the experimental procedure it was found that the robot has an adequately high repeatability and that the effective workspace, although with boundaries almost $30 \%$ more constrained than their theoretical values, is extensive and a clear advancement compared with that of current rigid tools. The repeatability of $0.42 \mathrm{~mm}$ achieved with our robotic end-effector is low when related to its workspace boundaries. This fact, combined with the very limited workspace of the endonasal approach and our intention for a handheld robotic tool where the surgeon is immediately controlling the robot and can thus provide visual feedback on the goal position, suggests adequate robot repeatability.

Additionally to these findings, and despite its miniature size, the robot presented promising structural integrity and was able to support and withstand a large amount of forces while maintaining a grasp on the load creating the force. These findings suggest that the end-effector developed in this study is feasible and we foresee it as the first implementation towards a handheld robotic tool suitable for the EEEA. Having said that, more development is required to address current limitations, starting with the replacement of the test-bench system used to validate the end-effector in this study, with an ergonomic handle to form the intended neurosurgical robotic tool mentioned in Section 1.

The development of this ergonomic handle is scope of future work, and our strategy is to develop a compact, ergonomic and intuitive instrument so that it is easily integrated into the surgical workflow, without a big learning curve that would discourage surgeons from using it. Compactness is especially important, because the instrument should allow simultaneous deployment with imaging modalities, such as a standard neurosurgical endoscope as proposed in the concept rendering of Fig. 1, or another neurosurgical tool. 

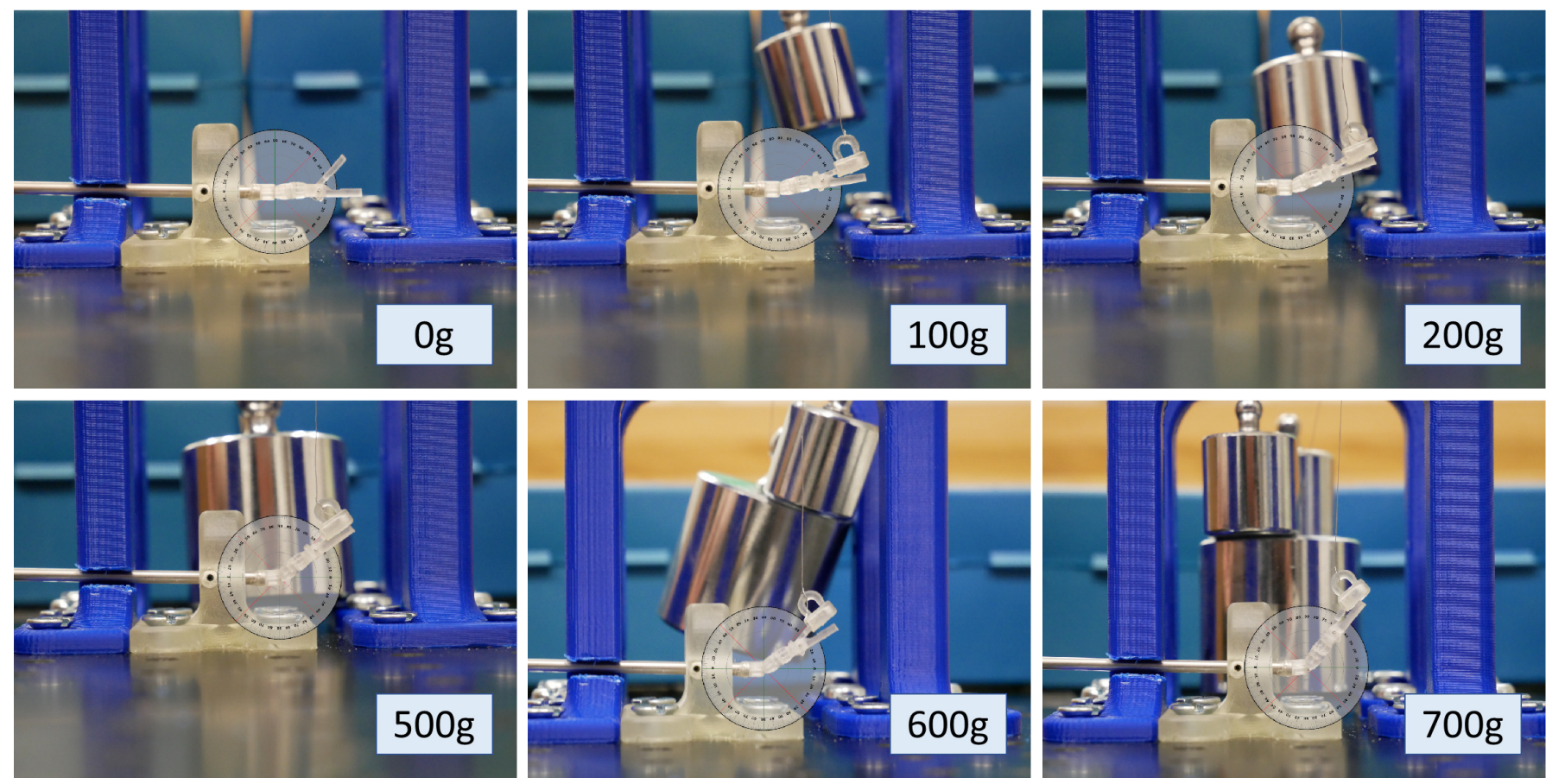

Fig. 16. (Left to right, top to bottom) The end-effector at its resting position, and supporting objects of $100 \mathrm{~g}, 200 \mathrm{~g}, 500 \mathrm{~g}, 600 \mathrm{~g}$ and $700 \mathrm{~g}$ mass. The deflection angle is also evident in the figure.

In our presented implementation, the joint-space is capped at an adequate but lower than expected $\pm 30^{\circ}$ range because we noticed that in movements beneath this threshold there was a small chance of tendon snapping. The nylon tendons used for this end-effector were not able to withstand the forces required to rotate the joints more than $35^{\circ}$, thus the movements were capped at a lower and safer value. Another limitation of this study is, of course, the difference between the theoretical workspace boundaries and the tracked ones. This, again, can be traced back to the tendons' elasticity and slipping. We also speculate that this inaccuracy could be a result of a less sophisticated motor control. When compared with other manipulators intended for the endonasal approach, ${ }^{24,},{ }^{28}$ the workspace is smaller, but when combined with the promising repeatability and structural integrity, the suggested end-effector could potentially suggest a substantial improvement over current instrumentation.

For the repeatability test, it is evident that the STD values for some of the joint-space angle vectors are abnormally large, especially the STD value for joint-space angle vector $[4,14]$ being $1.02 \mathrm{~mm}$. Such a large error could be attributed to various factors. There are some uncertainties concerning the implementation of the mechanism, namely tendon slacking resulting into an accumulated mechanical error, or kinematics simplifications resulting in a slight positioning error. Additionally, another factor we need to take into account is the possible measuring error that appears when the tracker has an unclear view of the markers.

Last but not least, during our structural integrity tests, although the results showed that the end-effector could avoid breakage and support a large amount of weight, a clear limitation appears in the large deflection angle created by the increasing load that would be a drawback during surgery. This deflection angle, that is relative to the weight of the object, could also be one of the reasons contributing to the end-effector's inability to maintain hold of the object attachment, as is evident in Fig. 16. However, the weight is most possibly still the main contributor since the deflection angle was the same for both the $500 \mathrm{gr}$ and $600 \mathrm{gr}$ objects, with only the $600 \mathrm{gr}$ object attachment slipping from the end-effector. Using a spherical joint requires careful tendon pre-tensioning since the joint and the base are not physically connected, as they would be in an articulated design. Thus, we suspect that the large deflection angle could be decreased with a better choice in tendons.

To avoid these challenges and to increase the workspace of the end-effector, we plan to investigate a variety of more durable tendon solutions and different tendon arrangements. Additionally, and before continuing with the development of the handheld tool, a more sophisticated tendon pre-tensioning strategy will be implemented by taking into account the tendon hysteresis to also try and compensate for the deflection caused by sudden forces. Clearly, the SLA printed robot end-effector will be replaced by metal parts, manufactured by either additive or subtractive manufacturing, properties such as stainless steel polishing and lubrication will be explored, and force-sensing will be integrated. 


\section{Conflict of Interest}

The authors declare that they have no conflict of interest.

\section{Acknowledgments}

This work was supported by the Wellcome/EPSRC Centre for Interventional and Surgical Sciences (WEISS) at UCL (203145Z/16/Z), EPSRC (EP/P027938/1, $\mathrm{EP} / \mathrm{R} 004080 / 1$ ), and the H2020 FET (GA 863146). Hani J. Marcus is supported by the National Brain Appeal, and the National Institute for Health Research, University College London Hospital Biomedical Research Centre. Danail Stoyanov is supported by a Royal Academy of Engineering Chair in Emerging Technologies (CiET1819 $\backslash 2 \backslash 36)$ and an EPSRC Early Career Research Fellowship (EP/P012841/1).

\section{References}

[1] B. S. Peters, P. R. Armijo, C. Krause, S. A. Choudhury, and D. Oleynikov, "Review of emerging surgical robotic technology," Surgical endoscopy, vol. 32, no. 4, pp. 1636-1655, 2018.

[2] H. J. Marcus, A. Hughes-Hallett, T. P. Cundy, D. Nandi, G.-Z. Yang, and A. Darzi, "Not everything that counts can be easily counted," $B m j$, vol. 346, p. f2461, 2013.

[3] C. S. Karas and E. A. Chiocca, "Neurosurgical robotics: a review of brain and spine applications," Journal of robotic surgery, vol. 1, no. 1, pp. 39-43, 2007.

[4] P. Cappabianca, L. M. Cavallo, and E. de Divitiis, "Endoscopic endonasal transsphenoidal surgery," Neurosurgery, vol. 55, no. 4, pp. 933-941, 2004.

[5] A. R. Dehdashti, A. Ganna, I. Witterick, and F. Gentili, "Expanded endoscopic endonasal approach for anterior cranial base and suprasellar lesions: indications and limitations," Neurosurgery, vol. 64, no. 4, pp. $677-$ 689, 2009.

[6] H. J. Marcus, T. P. Cundy, A. Hughes-Hallett, G.Z. Yang, A. Darzi, and D. Nandi, "Endoscopic and keyhole endoscope-assisted neurosurgical approaches: a qualitative survey on technical challenges and technological solutions," British journal of neurosurgery, vol. 28 , no. 5, pp. 606-610, 2014.

[7] J. Song, B. Gonenc, J. Guo, and I. Iordachita, "Intraocular snake integrated with the steady-hand eye robot for assisted retinal microsurgery," in 2017 IEEE International Conference on Robotics and Automation (ICRA), pp. 6724-6729, IEEE, 2017.

[8] J. Shang, D. P. Noonan, C. Payne, J. Clark, M. H. Sodergren, A. Darzi, and G.-Z. Yang, "An articulated universal joint based flexible access robot for minimally invasive surgery," in 2011 IEEE international conference on robotics and automation, pp. 1147-1152, IEEE, 2011.
[9] P. Berthet-Rayne, G. Gras, K. Leibrandt, P. Wisanuvej, A. Schmitz, C. A. Seneci, and G.-Z. Yang, "The i 2 snake robotic platform for endoscopic surgery," Annals of biomedical engineering, vol. 46, no. 10, pp. 1663$1675,2018$.

[10] J. Ding, R. E. Goldman, K. Xu, P. K. Allen, D. L. Fowler, and N. Simaan, "Design and coordination kinematics of an insertable robotic effectors platform for single-port access surgery," IEEE/ASME transactions on mechatronics, vol. 18, no. 5, pp. 1612-1624, 2012.

[11] F. Alambeigi, S. Sefati, R. J. Murphy, I. Iordachita, and M. Armand, "Design and characterization of a debriding tool in robot-assisted treatment of osteolysis," in 2016 IEEE International Conference on Robotics and Automation (ICRA), pp. 5664-5669, IEEE, 2016.

[12] A. L. Benabid, P. Cinquin, S. Lavalle, J. F. Le Bas, J. Demongeot, and J. De Rougemont, "Computerdriven robot for stereotactic surgery connected to CT scan and magnetic resonance imaging," Stereotactic and Functional Neurosurgery, vol. 50, no. 1-6, pp. 153154, 1987.

[13] M. S. Eljamel, "Validation of the PathFinder ${ }^{\top M}$ neurosurgical robot using a phantom," The International Journal of Medical Robotics and Computer Assisted Surgery, vol. 3, no. 4, pp. 372-377, 2007.

[14] H. J. Marcus, C. A. Seneci, C. J. Payne, D. Nandi, A. Darzi, and G.-Z. Yang, "Robotics in keyhole transcranial endoscope-assisted microsurgery: a critical review of existing systems and proposed specifications for new robotic platforms," Operative Neurosurgery, vol. 10, no. 1, pp. 84-96, 2013.

[15] H. J. Marcus, A. Hughes-Hallett, T. P. Cundy, G.Z. Yang, A. Darzi, and D. Nandi, "da Vinci robotassisted keyhole neurosurgery: a cadaver study on feasibility and safety," Neurosurgical review, vol. 38, no. 2, pp. 367-371, 2015.

[16] S. Chumnanvej, S. Chalongwongse, B. M. Pillai, and J. Suthakorn, "Pathway and workspace study of Endonasal Endoscopic Transsphenoidal (EET) approach in 80 cadavers," Elsevier International Journal of Surgery Open, vol. 16, pp. 22-28, 2019.

[17] C. Nimsky, J. Rachinger, H. Iro, and R. Fahlbusch, "Adaptation of a hexapod-based robotic system for extended endoscope-assisted transsphenoidal skull base surgery," min-Minimally Invasive Neurosurgery, vol. 47, no. 01, pp. 41-46, 2004.

[18] S. Chalongwongse and J. Suthakorn, "Workspace determination and robot design of a prototyped surgical robotic system based on a cadaveric study in Endonasal transsphenoidal surgery," in 2014 IEEE International Conference on Robotics and Biomimetics (ROBIO 2014), pp. 241-246, 2014.

[19] C. He, K. Olds, I. Iordachita, and R. Taylor, "A new ent microsurgery robot: error analysis and implementation," in 2013 IEEE International Conference on Robotics and Automation, pp. 1221-1227, 2013.

[20] J. Burgner, P. J. Swaney, D. C. Rucker, H. B. Gilbert, 
S. T. Nill, P. T. Russell, K. D. Weaver, and R. J. Webster, "A bimanual teleoperated system for endonasal skull base surgery," in 2011 IEEE/RSJ international conference on intelligent robots and systems, pp. 25172523, 2011.

[21] P. J. Swaney, J. M. Croom, J. Burgner, H. B. Gilbert, D. C. Rucker, R. J. Webster, K. D. Weaver, and P. T. Russell, "Design of a quadramanual robot for singlenostril skull base surgery," in ASME 2012 5th Annual Dynamic Systems and Control Conference joint with the JSME 2012 11th Motion and Vibration Conference, pp. 387-393, 2012.

[22] P. J. Swaney, H. B. Gilbert, R. J. Webster III, P. T. Russell III, and K. D. Weaver, "Endonasal skull base tumor removal using concentric tube continuum robots: a phantom study," Journal of Neurological Surgery Part B: Skull Base, vol. 76, no. 02, pp. 145149, 2015.

[23] J. Arata, Y. Tada, H. Kozuka, T. Wada, Y. Saito, N. Ikedo, Y. Hayashi, M. Fujii, Y. Kajita, and M. Mizuno, "Neurosurgical robotic system for brain tumor removal," International journal of computer assisted radiology and surgery, vol. 6, no. 3, pp. 375-385, 2011.

[24] J. Arata, Y. Fujisawa, R. Nakadate, K. Kiguchi, K. Harada, M. Mitsuishi, and M. Hashizume, "Compliant four degree-of-freedom manipulator with locally deformable elastic elements for minimally invasive surgery," in 2019 IEEE International Conference on Robotics and Automation (ICRA), pp. 2663-2669, 2019.

[25] G. Gerboni, P. W. J. Henselmans, E. A. Arkenbout, W. R. van Furth, and P. Breedveld, "HelixFlex: bioinspired maneuverable instrument for skull base surgery," Bioinspiration $\&$ biomimetics, vol. 10, no. 6, p. $66013,2015$.

[26] B. Zhang, Z. Liao, and H. Liao, "Visible forceps manipulator with novel linkage bending mechanism for neurosurgery," in 2017 39th Annual International Conference of the IEEE Engineering in Medicine and Biology Society (EMBC), pp. 4329-4332, IEEE, 2017.

[27] S. Kwon, W. Choi, G. Ryu, S. Kang, and K. Kim, "Endoscopic endonasal skull base surgery system," in 2017 14th International Conference on Ubiquitous Robots and Ambient Intelligence (URAI), pp. 544-545, IEEE, 2017.
[28] S.-i. Kwon, G. Ryu, S. Kang, and K. Kim, "A steerable endoscope for transnasal skull base surgery," in 2018 40th Annual International Conference of the IEEE Engineering in Medicine and Biology Society $(E M B C)$, pp. 4158-4161, IEEE, 2018.

[29] M. L. Guckert and M. D. Naish, "A compact 3 degree of freedom spherical joint," Journal of Mechanisms and Robotics, vol. 3, no. 3, 2011.

[30] A. Schmitz, S. Treratanakulchai, P. Berthet-Rayne, and G.-Z. Yang, "A Rolling-Tip Flexible Instrument for Minimally Invasive Surgery," in 2019 IEEE International Conference on Robotics and Automation (ICRA), pp. 379-385, 2019.

[31] D. Sareen, A. K. Agarwal, J. M. Kaul, and A. Sethi, "Study of sphenoid sinus anatomy in relation to endoscopic surgery," Int J Morphol, vol. 23, no. 3, pp. 261266, 2005.

[32] K. S. S. E. \&. C. KG, "All instruments for Endonasal Skull Base Surgery, Endoscopy and Microscopy,"

[33] F. Conti, F. Barbagli, R. Balaniuk, M. Halg, C. Lu, D. Morris, L. Sentis, J. Warren, O. Khatib, and K. Salisbury, "The CHAI libraries," in Proceedings of Eurohaptics 2003, pp. 496-500, 2003.

[34] E. Rohmer, S. P. N. Singh, and M. Freese, "Coppeliasim (formerly v-rep): a versatile and scalable robot simulation framework," in Proc. of The International Conference on Intelligent Robots and Systems (IROS), 2013. www.coppeliarobotics.com.

[35] N. Evangeliou, E. Dimitrakakis, and A. Tzes, "Design and experimental evaluation of a tendon-driven minimally invasive surgical robotic tool with antagonistic control," in 2017 IEEE Conference on Control Technology and Applications (CCTA), pp. 463-467, 2017.

[36] M. Quigley, K. Conley, B. Gerkey, J. Faust, T. Foote, J. Leibs, R. Wheeler, and A. Y. Ng, "Ros: an opensource robot operating system," in ICRA workshop on open source software, vol. 3, p. 5, 2009.

[37] B. KS, "7082: 1999 (iso 9283: 1998) robot peformance criteria," Association of Korean Standard, 1999.

[38] J. R. Bekeny, P. J. Swaney, R. J. Webster III, P. T. Russell, and K. D. Weaver, "Forces applied at the skull base during transnasal endoscopic transsphenoidal pituitary tumor excision," Journal of Neurological Surgery Part B: Skull Base, vol. 74, no. 06, pp. 337-341, 2013. 